\title{
Reinvestigation of the tautomerism of some substituted 2-hydroxypyridines
}

\author{
Luciano Forlani,* Giampiero Cristoni, Carla Boga, Paolo E. Todesco, Erminia Del \\ Vecchio, Simona Selva ${ }^{\S}$, and Magda Monari ${ }^{\S}$
}

Dipartimento di Chimica Organica "A. Mangini" viale Risorgimento 4, 40136 Bologna Italia

${ }^{\S}$ Dipartimento di Chimica “G. Ciamician”, via Selmi, 2, 40126, Bologna, Italia

E-mail: forlani@ms.fci.unibo.it

\section{Dedicated to Prof. Domenico Spinelli, on his $70^{\text {th }}$ birthday}

(received 28 Oct 02; accepted 19 Dec 02; published on the web 27 Dec 02)

\begin{abstract}
The tautomerism of some substituted 2-hydroxypyridines is investigated by UV/Vis- and ${ }^{1} \mathrm{H}-$, and ${ }^{13} \mathrm{C}$ - NMR spectroscopic methods, with the aid of the N-Me and O-Me fixed parents. NMR spectroscopic data do not allow discrimination between the two tautomeric forms (with the exception of the unsubstituted 2-hydroxypyridine), while UV/Vis- data permit the quantitative determination, in different solvents, of the amounts of the two forms. The electronic substituent effect and the change of solvent are discussed. An X-Ray diffraction study carried out on a crystal of 2-hydroxy-5-nitropyridine (7) reveals that this compound, in the solid state, is in the oxo-form.
\end{abstract}

Keywords: Hydroxypyridine, tautomerism, electronic effect of substituents, X-ray diffraction

\section{Introduction}

Our interest is in the prototropic equilibrium in heterocyclic aza- containing series ${ }^{1}$ as shown in Scheme 1.

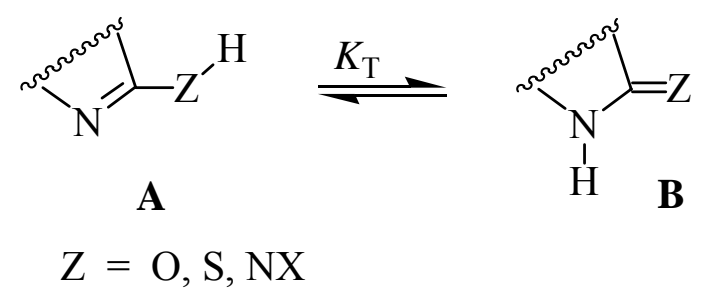

\section{Scheme 1}


Careful attention has been devoted to the tautomeric equilibrium of Scheme 2 and, in particular, to the electronic properties of the group $\mathrm{X}$ bonded to the exocyclic nitrogen. ${ }^{2}$ The addition of a small amount of polar substances to solutions of some 2-acetamidothiazoles shifts the equilibrium of Scheme 2 from the amido aromatic form A (the most stable in apolar solvents) to the imido form $\mathbf{B}$.

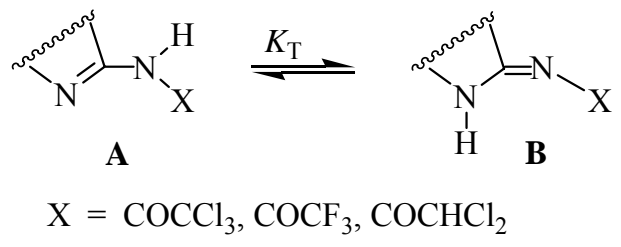

\section{Scheme 2}

In the six-membered ring series (pyrimidine, pyridine) the amino aromatic tautomer is (in every case) the predominant species, while in the penta-atomic series (1,3-thiazole) when $\mathrm{X}$ is a strong electron-withdrawing group, form $\mathbf{B}$ may predominate, depending on the polarity of the medium..$^{2-4}$ In the thiazole series, the position of the tautomeric equilibrium of Scheme 2 is scarcely affected by change of the substituent on the thiazole moiety.

The continuing interest in the hydroxypyridines 1-3 (Scheme 3) arises from the fact that these simple compounds may be used as models to explain the behavior of more complicated systems such as nucleic acid bases.

Recently, we reported data on the reactions of the three isomeric hydroxypyridines ${ }^{5}$ and of cytosine, thymine and uracil $^{6}$ toward 1-chloro-2,4,6-trinitrobenzene. In some cases, the electronic interactions between the hydroxy- and aza- group may explain the reactivity and the regioselectivity of the reactions of these compounds with the electrophilic reagent used.
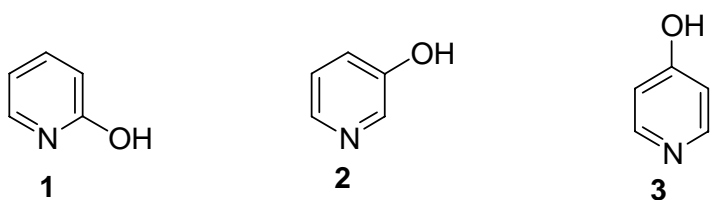

\section{Scheme 3}

Among the three isomeric hydroxypyridines $1-3$, the reactivity and tautomeric properties of 2-hydroxypyridine (1) have been most studied.

In general, ${ }^{7}$ it is known that 2-hydroxypyridine, in the condensed phase, is in an oxo-amido form $\mathbf{B}$, and is in the imido aromatic form $\mathbf{A}$ in dilute gas phase, as shown in the equilibrium of Scheme 1. However, when particular substituents are bonded to the pyridine ring, the form $\mathbf{B}$ may be the more populated form in the condensed phase too. Kinetic investigations on some 
substituted 2-hydroxypyridines in solvents under experimental conditions which favor the contemporaneous presence of both tautomers have been reported..$^{8-11}$

In order to collect further information on the influence of substituents bonded to the heterocyclic ring, upon the position of the tautomeric equilibrium, we report investigations performed by UV/Vis- and ${ }^{1} \mathrm{H}$ - and ${ }^{13} \mathrm{C}$ - NMR spectroscopic methods of some 2hydroxypyridines and of their fixed parents (Scheme 4). The effect of the change of the substituent on the position of the tautomeric equilibrium of Scheme 4 is also investigated in different solvents, including polar, apolar, protic, and aprotic solvents (DMSO, THF, MeOH, $\mathrm{CH}_{2} \mathrm{Cl}_{2}, \mathrm{CCl}_{4}$ ). Some solvent/water mixtures are also investigated.

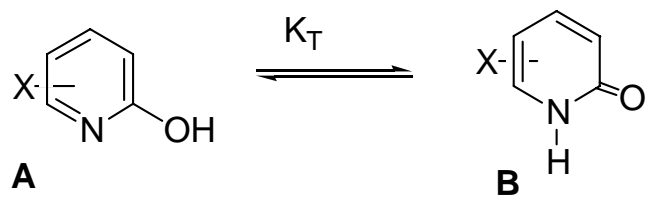

$$
\mathrm{X}=\mathrm{H}(\mathbf{1}), 6-\mathrm{Cl}(\mathbf{4}), 6-\mathrm{NH}_{2}(\mathbf{5}), 6-\mathrm{OCH}_{3}(\mathbf{6}), 5-\mathrm{NO}_{2}(\mathbf{7})
$$

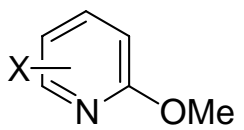

$$
\begin{aligned}
& \mathrm{X}=\mathrm{H}(1-\mathrm{OMe}), 6-\mathrm{Cl}(4-\mathrm{OMe}), 6-\mathrm{OCH}_{3}(6-\mathrm{OMe}), 5-\mathrm{NO}_{2} \text { (7-OMe) } \\
& \mathrm{X}=\mathrm{H}(1-\mathrm{NMe}), 6-\mathrm{Cl}(4-\mathrm{NMe}), 6-\mathrm{OCH}_{3}(6-\mathrm{NMe}), 5-\mathrm{NO}_{2}(7-\mathrm{NMe})
\end{aligned}
$$

\section{Scheme 4}

Particular attention was devoted to 2-hydroxy-5-nitropyridine (7), on which a few data are in the literature. The crystal structure of 7 has also been ascertained by X-ray diffraction analysis.

\section{Results and Discussion}

\section{${ }^{1} \mathrm{H}$ - and ${ }^{13} \mathrm{C}$ - NMR spectroscopic data}

To obtain information on the relative population of tautomers of the considered hydroxypyridines, we report NMR spectral data of compounds 1,4,6,7 and of the related "fixed 
parents" in a weakly polar solvent $\left(\mathrm{CDCl}_{3}\right)$ and in a polar solvent $\left(\mathrm{DMSO}-\mathrm{d}_{6}\right)$. The data are collected in Tables 7 and 8 in the Experimental section, and they agree with available literature data (see references cited in Tables 7,8).

Some main considerations worthy of attention are as follows:

1) In agreement with Philipsborn's reports, ${ }^{12}$ compound $\mathbf{1}$ is mainly in the oxo- form $\mathbf{B}$ in both solvents.

2) 2-Hydroxy-6-chloropyridine (4) and 2-hydroxy-6-methoxypyridine (6) are probably in form A in both solvents $\left(\mathrm{CDCl}_{3}\right.$ and $\left.\mathrm{DMSO}\right)$ because they show proton signals similar to those of the OMe derivative, but the differences are too weak to discriminate with certainty between the two forms.

3) Both the ${ }^{1} \mathrm{H}$ - and ${ }^{13} \mathrm{C}$ - NMR signals of 2-hydroxy-5-nitropyridine (7) in DMSO-d $\mathrm{d}_{6}$ and in $\mathrm{CD}_{3} \mathrm{OD}$ indicate that $\mathbf{7}$ is mainly in form $\mathbf{B}$. However, the spectroscopic data of 7 in DMSO- $\mathrm{d}_{6}$ and in the presence of an excess of DABCO, are similar to that of compound 7-NMe. As confirmed by UV/Vis- spectroscopic data, the presence of the anionic form of 7 complicates the spectral analysis. In $\mathrm{CDCl}_{3}$ the NMR spectroscopic data cannot discriminate between form $\mathbf{A}$ and B.

\section{UV/Vis- spectroscopic data}

To evaluate the position of the tautomeric equilibrium of Scheme 4 we recorded the UV/Visspectra of compounds $1,4,5,6,7$ and of the related methyl derivatives, where the methyl group is bonded to the endocyclic nitrogen or to the exocyclic oxygen atom of the potential OH group. Spectroscopic data are reported in the Experimental Section.

It is of interest to observe that for all the compounds considered, i.e., both the potential tautomers and the fixed parents, and for all the solvents considered, the Lambert-Beer law is followed well in the concentration range from $2 \times 10^{-5}$ to $2 \times 0^{-4}$. Hydroxypyridines are well known to have a self-association (dimerization) equilibrium. ${ }^{13}$ In the range of concentration values used here, the possible dimer formation does not influence the spectroscopic data.

From the analytical data, $K_{\text {Tapp }}$ are calculated by using literature methods, ${ }^{14}$ and are reported in Table 1.

Table 1. $\log K_{\text {Tapp }}$ of the considered compounds, in a number of solvents

\begin{tabular}{|c|c|c|c|c|c|}
\hline \multirow[t]{2}{*}{ Solvent $^{\mathrm{a}}$} & \multicolumn{5}{|c|}{$\log K_{\text {Tapp }}$} \\
\hline & 1 & 4 & 5 & 6 & 7 \\
\hline THF (37.4) & 0.800 & -1.778 & $-0.278^{b}$ & -1.193 & -2.310 \\
\hline $\mathrm{CH}_{2} \mathrm{Cl}_{2}(41.1)$ & - & - & - & - & -1.990 \\
\hline $\mathrm{CCl}_{4}(32.5)$ & 0.950 & -1.302 & - & -1.053 & -1.611 \\
\hline DMSO (45.0) & 1.121 & -1.176 & - & -0.607 & - \\
\hline
\end{tabular}


Table 1. Continued

\begin{tabular}{llllll}
\hline $\mathrm{CH}_{3} \mathrm{OH}(55.5)$ & 1.570 & -0.464 & - & 0.403 & -1.031 \\
& & & & & \\
$\mathrm{H}_{2} \mathrm{O}(63.1)$ & 1.960 & 0.339 & - & 1.166 & -0.338 \\
\hline
\end{tabular}

${ }^{\mathrm{a}} \mathrm{E}_{\mathrm{T}}$ values from Reichardt, C. Solvents and Solvent Effects in Organic Chemistry, Verlag Chemie: Weinheim, 1988.

${ }^{\mathrm{b}}$ From Fujimoto, A.; Inozuka, K. Bull. Chem. Soc. Jpn. 1990, 63, 2292.

Some data in the literature ${ }^{15}$ agree with the present data, but some others disagree, probably because of the difference in method of obtaining $K_{\mathrm{T}}$ values.

$$
K_{\text {Tapp }}=[\text { lactam }] /[\text { lactim }]=[\mathbf{B}] /[\mathbf{A}]
$$

In order to obtain information on the influence of added protic solvent, we checked the variations of tautomeric equilibrium in THF and in DMSO, by adding $\mathrm{H}_{2} \mathrm{O}$. For both hydroxypyridines 4 and $\mathbf{6}$, the addition of $\mathrm{H}_{2} \mathrm{O}$ to THF (or DMSO) solutions shifts the ratio $[\mathbf{B}] /[\mathbf{A}]$ toward the form $\mathbf{B}$, as reported in Table 2 .

Table 2. $\log K_{\mathrm{T}}$ values of 2-hydroxypyridines, at $20^{\circ} \mathrm{C}$, in mixtures of solvent with $\mathrm{H}_{2} \mathrm{O} . \mathrm{X}_{\mathrm{H}_{2} \mathrm{O}}$ is the molar fraction of water.

2-Hydroxy-6-chloropyridine (4), solvent $=\mathrm{THF}$

\begin{tabular}{ccccccccccccc}
\hline $\mathrm{x}_{\mathrm{H}_{2} \mathrm{O}}$ & 0.0 & 0.33 & 0.47 & 0.62 & 0.69 & 0.78 & 0.82 & 0.84 & 0.87 & 0.89 & 0.90 & 0.91 \\
$\log K_{\mathrm{T}}$ & -1.778 & -1.243 & -1.080 & -0.879 & -0.743 & -0.537 & -0.414 & -0.317 & -0.165 & -0.099 & -0.017 & 0.050 \\
$\mathrm{x}_{\mathrm{H}_{2} \mathrm{O}}$ & 0.94 & 0.96 & 0.98 & 1.0 & & & & & & & & \\
$\log K_{\mathrm{T}}$ & 0.199 & 0.275 & 0.328 & 0.339 & & & & & & & & \\
\hline
\end{tabular}

2-Hydroxy-6-methoxypyridine (6), solvent $=\mathrm{THF}$

\begin{tabular}{ccccccccccccc}
\hline $\mathrm{X}_{\mathrm{H}_{2} \mathrm{O}}$ & 0.0 & 0.13 & 0.24 & 0.33 & 0.47 & 0.62 & 0.69 & 0.78 & 0.82 & 0.90 & 0.98 & 1.0 \\
$\log K_{\mathrm{T}}$ & -1.193 & -0.918 & -0.649 & -0.498 & -0.253 & 0.053 & 0.161 & 0.530 & 0.719 & 0.927 & 1.107 & 1.166 \\
\hline
\end{tabular}

2-hydroxy-6-methoxypyridine (6); solvent = DMSO

\begin{tabular}{ccccccccccc}
\hline $\mathrm{x}_{\mathrm{H}_{2} \mathrm{O}}$ & 0.0 & 0.13 & 0.35 & 0.44 & 0.52 & 0.65 & 0.71 & 0.76 & 0.81 & 1.0 \\
$\log K_{\mathrm{T}}$ & -0.607 & -0.583 & -0.363 & -0.239 & -0.089 & 0.231 & 0.378 & 0.534 & 0.703 & 1.166 \\
\hline
\end{tabular}

In agreement with the data in Table 2, and with some literature reports, ${ }^{8,11}$ in all cases the ratio $[\mathbf{B}] /[\mathbf{A}]$ increases on increasing the amount of water. The $[\mathbf{B}] /[\mathbf{A}]$ ratio is higher in water than in DMSO, or in THF, or in $\mathrm{CCl}_{4}$. This fact agrees with the data of Table 1 . In fact, by 
considering the medium's polarity, as expressed by Dimroth's parameter, $\mathrm{E}_{\mathrm{T}}$, the plot of Figure 1 shows that the protic solvent does not cause any peculiarity with respect to the aprotic solvent.

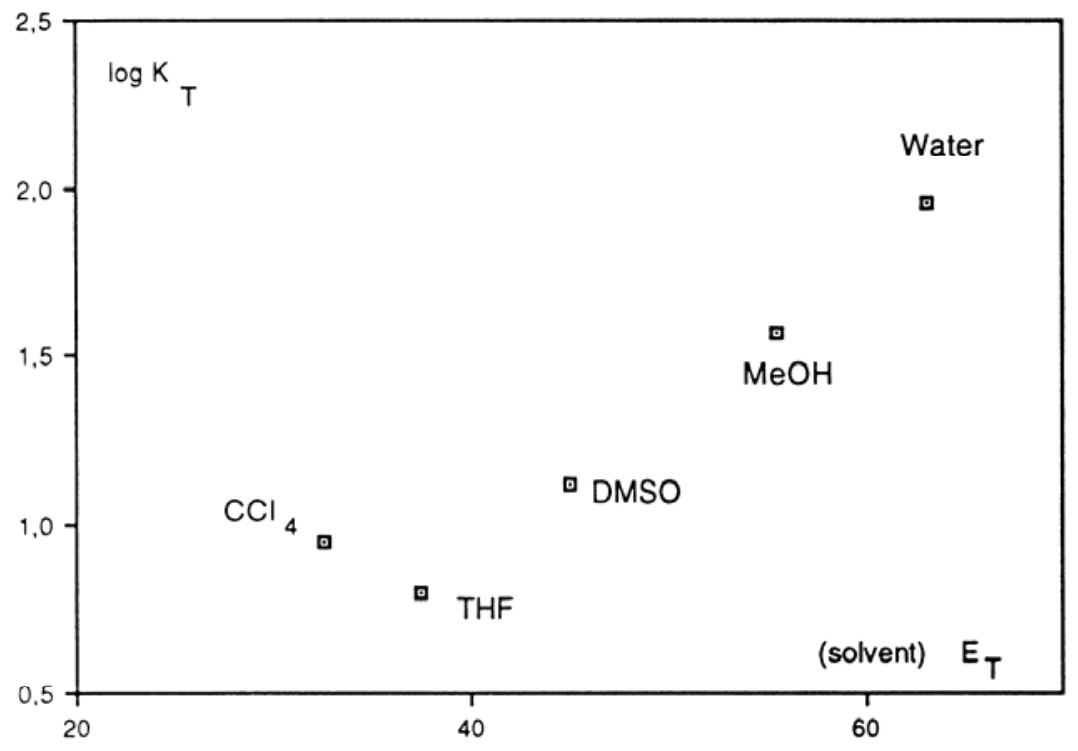

Figure 1. Log $\mathrm{K}_{\mathrm{T}}$ values versus Dimroth solvent parameters.

The main parameter to determine the position of the tautomeric equilibrium appears to be the medium's polarity and not its proticity. This behavior disagrees with a possible ${ }^{9}$ pathway for the tautomeric displacement involving the solvent's proton-donor ability. For all the hydroxypyridines considered, $\mathrm{CCl}_{4}$ deviates from the linearity. Probably, for this solvent, which is highly associating, the dimer mechanism is an important pathway to the tautomerism because the "dimer's" presence is indicated to favor the form $\mathbf{B} .{ }^{10}$

Usually, the dimer is present more in a more associating solvent: thus $\mathrm{CCl}_{4}$ favors the selfassociation of 2-hydroxypyridines more than THF, DMSO, and water. DMSO and water do not favor self-association of $\mathbf{1}$ and of related compounds, with the exception of the model compounds of course. In these solvents the form $\mathbf{B}$ is more populated than form A, indicating that another mechanism, different from the dimer mechanism (Scheme 5), may operate in these solvents.

Probably, other parameters, such as the ability of the solvent to have hydrogen-bonding interactions, operate in stabilizing a tautomeric form.

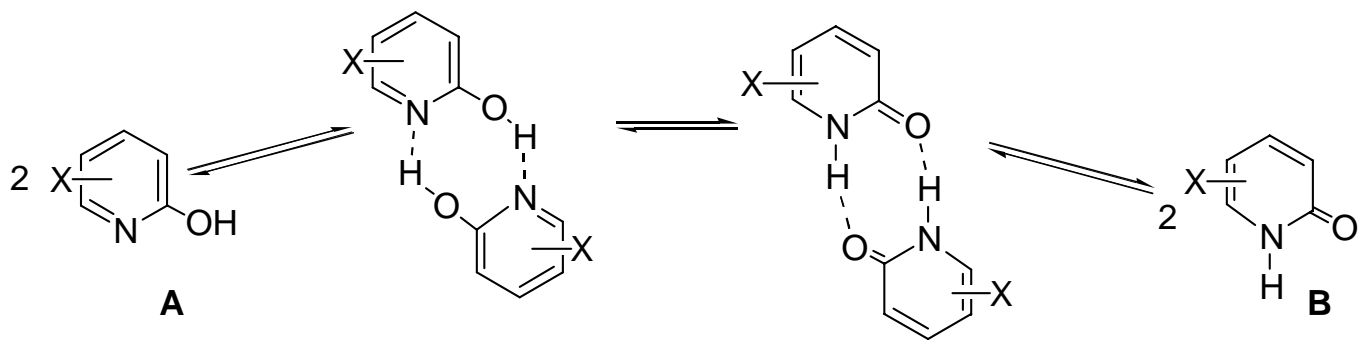




\section{Scheme 5}

\section{Electronic effect of substituents and solvent change}

Even if the number of substituents used here does not allow a full data dissection, some indications are worthy of consideration. For all solvents, the best fit of data of Table 1 in the Hammett treatment of the electronic effect of substituents, is given by using the substituent constant $\sigma_{\mathrm{I} .}{ }^{16} \tilde{\rho}$ Values for the solvents considered are high and negative (see Table 3 ).

Table 3. $\rho$ values from the Hammett equation by using the $\sigma_{\mathrm{I}}$ values and $\log K_{\mathrm{T}}$ values of Table 1

\begin{tabular}{ccc}
\hline Solvent &.$\rho^{\mathrm{a}}$ & $\mathrm{R}^{\mathrm{b}}$ \\
\hline $\mathrm{THF}$ & $-4.6 \pm 0.7$ & 0.964 \\
$\mathrm{CCl}_{4}$ & $-4.0 \pm 1$ & 0.917 \\
$\mathrm{DMSO}$ & $-5.0 \pm 1$ & 0.9702 \\
$\mathrm{CH}_{3} \mathrm{OH}$ & $-4.1 \pm 0.2$ & 0.998 \\
$\mathrm{H}_{2} \mathrm{O}$ & $-3.6 \pm 0.2$ & 0.998 \\
\hline
\end{tabular}

${ }^{a}$ Errors are standard deviations.

${ }^{\mathrm{b}}$ Correlation coefficient.

In principle, the use of $\sigma_{I}$ values and the calculated $\rho$ values may be explained in two main ways.

(a) The substituents mainly affect the acidity of the $\mathrm{O}-\mathrm{H}$ bond which influence the position of the equilibrium.

Position 6 of the pyridine ring is a meta-like position with respect to position 2 bearing the $\mathrm{O}-\mathrm{H}$ group. Consequently, from the group in position 6 to the group in position 2 the main electronic effect is inductive in character, in agreement with the use of $\sigma_{\mathrm{I}}$ values. This explanation does not agree with the use of the $\sigma_{I}$ value of the nitro group bonded on position 5 , because position 5 is para-like with respect to position 2 , but it is meta-like with respect to the "aza" nitrogen.

(b) The substituent effect mainly acts on the N-H bond.

When the protonation equilibrium of 2 -substituted pyridines ${ }^{17}$ (or of other related "aza"containing heterocycles) ${ }^{18}$ is considered, as reported in Scheme 6 , the best fit is obtained by using $\sigma_{\text {meta }}$ values. In these cases the $\rho$ value is very high and negative $(\rho=-11.8)$.

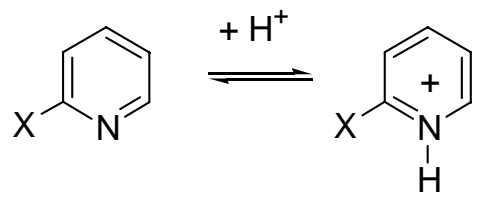

\section{Scheme 6}


The high $\rho$ value is explained by the fact that the reaction center of the equilibrium of Scheme 6 (the "aza" nitrogen) and the 2- substituent are bonded to the same carbon atom in the heterocyclic ring.

Clearly, the $\rho$ values reported here are much lower than that required for the simple protonation shown in Scheme 6.

For the same substituents considered here, the Hammett plot of $\mathrm{p} K_{\mathrm{a}}$ values of 2hydroxypyridines (see Scheme 7) vs. $\sigma_{\mathrm{I}}$ values produces the $\rho$ values of $-(8.2 \pm 0.3) ;\left(\mathrm{p} K_{\mathrm{a}}\right.$ values $^{9,19}$ of $\mathbf{1}, 2$-hydroxy-6-methylpyridine, $\mathbf{4 ,} \mathbf{6}, \mathbf{7}=11.70,12.45,7.84,9.82,6.85$, respectively).

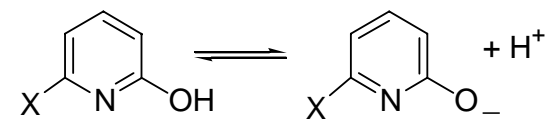

\section{Scheme 7}

Probably, the $\rho$ value of -4 arises from a balance of two main effects; the acidity of the N-H bond of form $\mathbf{B}$ and (less relevant, because further away from the substituent) the proton affinity of the oxygen atom to yield form A. This conclusion supports the "dimer" mechanism, as reported in Scheme 5.

Of course, it is not necessary to involve a cyclic dimeric form, but the bimolecular proton transfer may occur by an equilibrium, as shown in Scheme 8.

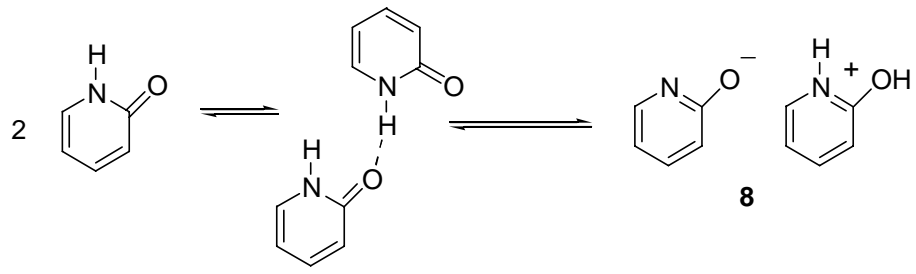

\section{Scheme 8}

In the proton transfer of Scheme 8, the intervention of the solvent may occur in two ways, by substituting the proton acceptor molecule or (when polar) by stabilizing the ion pairs 8.

The variations in the $\rho$ value observed when the solvent is changed are slightly higher than the errors, calculated as standard deviation (mean $4 \pm 0.6$, range 1.4 ). This is an indication that the importance of the electronic (inductive) effect of the substituent is similar for all the solvents considered. With respect to the solvent, the $\rho$ values may be assessed on the scale DMSO $>\mathrm{THF}>\mathrm{CCl}_{4}, \mathrm{CH}_{3} \mathrm{OH}>\mathrm{H}_{2} \mathrm{O}$. The electron-withdrawing ability of substituents is more important in stabilizing form $\mathbf{B}$ in DMSO than in water. This fact confirms that the solvent polarity is the main parameter in stabilizing form B. Other possible parameters such as the proton-donor ability of the solvent are probably of little importance. 
We emphasize that the change of solvent involves polar protic solvents (water, methanol), polar aprotic (dimethyl sulfoxide), poorly polar (tetrahydrofuran) and apolar solvents (carbon tetrachloride and dichloromethane). Electron-withdrawing substituents clearly (strongly) favor the lactim form A. Further evidence for the preferred stability of lactim forms comes from computational studies. ${ }^{20}$

\section{Effect of changes of temperature}

Table 4 reports the effect of the changes of the temperature on $[\mathbf{B}] /[\mathbf{A}]$ ratios in a mixed solvent $\left(\mathrm{THF} / \mathrm{H}_{2} \mathrm{O}\right)$. The spectroscopic data obtained at different temperatures, for compounds $\mathbf{4}$ and $\mathbf{6}$ in $\mathrm{THF} /$ water mixtures indicate that the equilibrium is strongly affected by changes in temperature. An increase in temperature produces an increase of the species $\mathbf{A}$ and a decrease of species $\mathbf{B}$.

Table 4. $\log K_{\mathrm{T}}$ values, at different temperatures, of 2-hydroxypyridines in mixtures $\mathrm{THF} / \mathrm{H}_{2} \mathrm{O}$. 2-Hydroxy-6-chloropyridine (4); $\mathrm{X}_{\mathrm{H} 2 \mathrm{O}}=0.98$

\begin{tabular}{lllllll}
\hline $\mathrm{T}\left({ }^{\circ} \mathrm{C}\right)$ & 15 & 20 & 24 & 29 & 35 & 40 \\
$\log K_{\mathrm{T}}$ & 0.364 & 0.328 & 0.305 & 0.269 & 0.248 & 0.221
\end{tabular}

2-Hydroxy-6-methoxypyridine (6) $\mathrm{X}_{\mathrm{H} 2 \mathrm{O}}=0.69$.

\begin{tabular}{lllllll}
\hline $\mathrm{T}\left({ }^{\circ} \mathrm{C}\right)$ & 20 & 25 & 31 & 34 & 40 & 45 \\
$\log K_{\mathrm{T}}$ & 0.161 & 0.140 & 0.127 & 0.114 & 0.093 & 0.072
\end{tabular}

In the case of 2-acetamidothiazoles, we reported ${ }^{4}$ that this behavior applies independently from the used substrates, and for all the added polar solutes. The present data agree with this conclusion, which may be considered of general application.

Table 4 reports data on the effect of the changes of the temperature on the $K_{\mathrm{T}}$ values: this behavior agrees with literature data. ${ }^{8-12}$ From the data of Table 4 it is possible to calculate the thermodynamic parameters, $-\Delta \mathrm{H}\left(\mathrm{kJ} \mathrm{mol}^{-1}\right)(6.2 \pm 0.1)$ and $(9.7 \pm 0.3),-\Delta \mathrm{S}\left(\mathrm{J} \mathrm{mol}^{-1} \mathrm{~K}^{-1}\right)(23 \pm 0.1)$ and $(34 \pm 0.3)$ for hydroxypyridines 6 and $\mathbf{4}$, respectively [errors are calculated as standard deviation]. The $\Delta \mathrm{S}$ values agree with a multi-step mechanism, such as the associating pathways of Schemes 7 and 8.

\section{2-Hydroxy-5-nitropyridine (7)}

Compound 7 deserves some consideration. The ${ }^{1} \mathrm{H}$ - and ${ }^{13} \mathrm{C}-\mathrm{NMR}$ data in $\mathrm{CDCl}_{3}$, DMSO- $\mathrm{d}_{6}$ and in $\mathrm{MeOH}-\mathrm{d}_{4}$ are poorly indicative of the predominance of one tautomeric form. Weak differences between the fixed O-Me, N-Me parents, and 7 (see Experimental Section) in the NMR spectroscopic data are recorded. The dissociation of the $\mathrm{O}-\mathrm{H}$ bond, in the equilibrium of Scheme 9 , complicates the diagnostic effort for this compound.

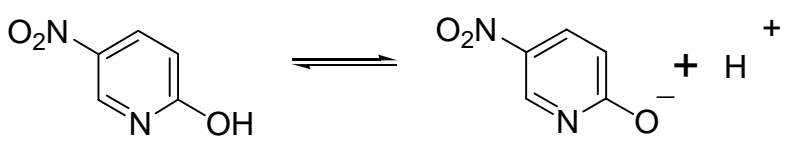

\section{Scheme 9}


The ${ }^{1} \mathrm{H}$ - and ${ }^{13} \mathrm{C}$ - NMR data in DMSO- $\mathrm{d}_{6}$ and in the presence of 1,4-diaza-[2,2,2]-bicyclooctane $(\mathrm{DABCO})$ fall in the same region as the fixed parents.

UV/vis. spectroscopic data in DMSO clearly indicate the presence of the anionic form of 7 $\left(\lambda_{\max }=342 \mathrm{~nm}, \varepsilon=760\right)$. Addition of DABCO enhances this absorbance maximum, which is absent in other solvents.

NOE experiments performed by irradiating the N-H signal of a solution of 7 in DMSO- $\mathrm{d}_{6}$ show an enhancement of the H-6 signal, indicating the proximity of H-6 to the acidic proton. This fact agrees with the tautomer 7B (Scheme 10) or with the dimer 7C (Scheme 11). The presence of a 7D-like specie, arising from self-protonation equilibrium, is hardly probable under our experimental conditions. Attempts to perform NOE experiments in other solvents failed.

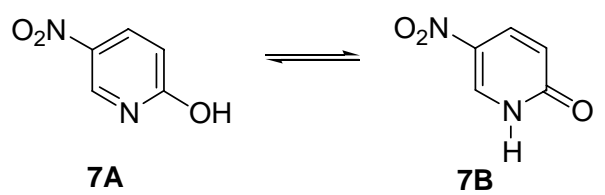

Scheme 10
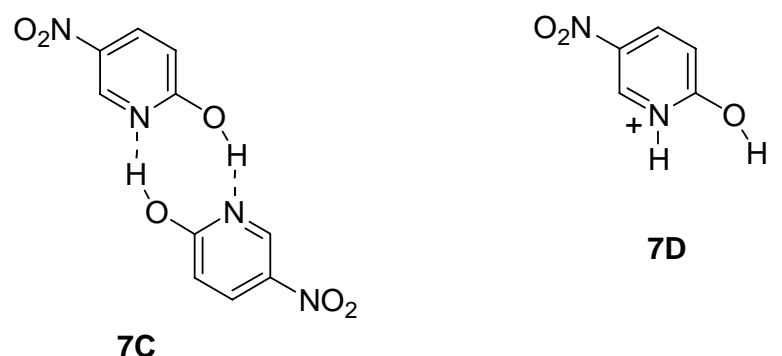

\section{Scheme 11}

\section{Molecular structure of 7}

The molecular structure of 2-hydroxy-5-nitropyridine (7) is shown in Figure 2 and relevant bond lengths and angles are reported in Table 5.

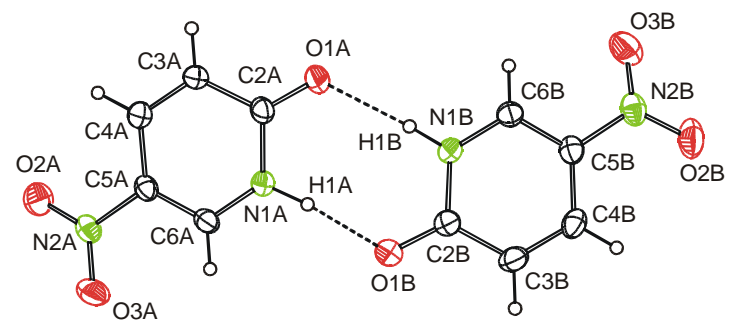

Figure 2. ORTEP drawing of 2-hydroxy-5-nitropyridine (7) (thermal ellipsoids at the 30\% probability level) showing the dimer held together by $\mathrm{N}-\mathrm{H} \cdot \mathrm{O}$ hydrogen bonds. 
In the solid state, two independent molecules are held together via one pair of $\mathrm{N}-\mathrm{H}$...O hydrogen bonds [O1A...H1B 1.96, O1B...H1A 1.80(4) Å, O1A-H1B-N1B 175(3) ${ }^{\circ}$ and O1BH1A-N1A $174(3)^{\circ}$ ], forming a dimer. The two molecules of the dimer are planar but not parallel to one another [dihedral angle between the planes of the molecules $=22.8(1)^{\circ}$ ] and as a consequence of this misalignment the dimer is quasi-centrosymmetric. The intramolecular distances are similar in the two molecules, and are closely related to those reported for 2hydroxypyridine $^{21}$ (Table 6) and 2-hydroxy-5-chloropyridine ${ }^{22 a}$ which appears in the keto form. The C-C distances show a considerable degree of variability and fall in the range 1.342-1.428(4) $\AA$ and $1.350-1.425(4) \AA$ in $\mathbf{A}$ and $\mathbf{B}$, respectively. The C3-C4 and C5-C6 interactions [C3-C4 1.352 and 1.350(4) $\AA$; C5-C6 1.342 and 1.356(4) $\AA$ in $\mathbf{A}$ and B] have a double- bond character whereas in the $\mathrm{C} 4-\mathrm{C} 5$ and $\mathrm{C} 3-\mathrm{C} 4$ bonds [C4-C5 1.411 and 1.403(4) $\AA$; C2-C3 1.428 and $1.425(4) \AA$ in $\mathbf{A}$ and $\mathbf{B}$ ] the $\pi$ - bonding contribution is significantly smaller. The pyridine nitrogen is involved in a short interaction with C(6) [N1-C6 1.345 and 1.352(4) $\AA$ ] and a longer one with the carbon [C2] of the electron-withdrawing carbonyl group [N1-C2 1.394 and 1.374(4) $\AA$ in A and B]. The $\mathrm{C}=\mathrm{O}$ distance [C2-O1 1.238 and 1.245(4) $\AA$ in A and B] is slightly shorter than that reported for 2-pyridone ${ }^{21}$ (Table 6). A comparison of the relevant bond lengths among 7, 2-pyridone and 2-hydroxy-6-chloropyridine ${ }^{2 \mathrm{~b}}$ (Table 6) shows that in the latter compound's adopting the enol form the $\mathrm{C} 2-\mathrm{O} 1$ bond is significantly longer than in 7 and 2pyridone. Conversely the $\mathrm{C} 2-\mathrm{N} 1$ interaction in 2-hydroxy-6-chloropyridine is shorter than in 7 and 2-pyridone. The $\mathrm{C}-\mathrm{NO}_{2}$ interaction [C5-N2 1.448 and 1.445(4) $\AA$ has a value typical for a $\mathrm{N}\left(\mathrm{sp}^{2}\right)-\mathrm{C}\left(\mathrm{sp}^{2}\right)$ bond. Interestingly, the nitro group is tilted away from the plane of the 2hydroxypyridine ring by $9.4(3)^{\circ}\left[11.8(3)^{\circ}\right.$ in $\left.\mathbf{B}\right]$.

Table 5. Bond lengths $[\AA]$ and angles $\left[{ }^{\circ}\right]$ for 2-hydroxy-5-nitropyridine (7) (distances and angles for molecule $\mathbf{B}$ are given in square brackets)

$\begin{array}{llll}\mathrm{N}(1)-\mathrm{H}(1) & 0.95(3)[0.90(3)] & \mathrm{N}(1)-\mathrm{C}(6) & 1.345(4)[1.352(4)] \\ \mathrm{H}(1 \mathrm{~A}) \cdots \mathrm{O}(1 \mathrm{~B}) & 1.80(4) & \mathrm{O}(1)-\mathrm{C}(2) & 1.231(3)[1.245(4)] \\ \mathrm{H}(1 \mathrm{~B}) \cdots \mathrm{O}(1 \mathrm{~A}) & 1.96(4) & & \\ \mathrm{N}(1 \mathrm{~A}) \cdots \mathrm{O}(1 \mathrm{~B}) & 2.750(4) & \mathrm{C}(2)-\mathrm{C}(3) & 1.428(4)[1.425(4)] \\ \mathrm{N}(1 \mathrm{~B}) \cdots \mathrm{O}(1 \mathrm{~A}) & 2.854(3) & & 1.352(4)[1.350(5)] \\ \mathrm{N}(2)-\mathrm{C}(5) & 1.448(4)[1.445(4)] & \mathrm{C}(3)-\mathrm{C}(4) & 1.411(4)[1.403(5)] \\ \mathrm{N}(2)-\mathrm{O}(2) & 1.212(3)[1.226(4)] & \mathrm{C}(4)-\mathrm{C}(5) & 1.342(4)[1.356(4)] \\ \mathrm{N}(2)-\mathrm{O}(3) & 1.221(4)[1.228(4)] & \mathrm{C}(5)-\mathrm{C}(6) & \\ \mathrm{N}(1)-\mathrm{C}(2) & 1.394(4)[1.374(4)] & & \end{array}$


Table 5. Continued

\begin{tabular}{llll}
\hline $\mathrm{N}(1 \mathrm{~A})-\mathrm{H}(1 \mathrm{~A}) \cdots \mathrm{O}(1 \mathrm{~B})$ & $174(3)$ & $\mathrm{C}(6)-\mathrm{N}(1)-\mathrm{H}(1)$ & $123(2)[119(2)]$ \\
$\mathrm{N}(1 \mathrm{~B})-\mathrm{H}(1 \mathrm{~B}) \cdots \mathrm{O}(1 \mathrm{~A})$ & $175(3)$ & & \\
$\mathrm{O}(1)-\mathrm{C}(2)-\mathrm{C}(3)$ & $125.6(3)[125.0(3)]$ & $\mathrm{C}(2)-\mathrm{N}(1)-\mathrm{H}(1)$ & $113(2)[117(2)]$ \\
$\mathrm{O}(1)-\mathrm{C}(2)-\mathrm{N}(1)$ & $119.3(3)[119.5(3)]$ & & \\
\hline
\end{tabular}

Table 6. Selected intra- and inter-molecular distances $(\AA)$ and assigned structure in crystals of compounds 7, 1 and 4.

\begin{tabular}{llll} 
& Ref. 22 \\
\hline $\mathrm{C}(2)-\mathrm{O}(1)$ & $1.238(4)$ & $1.251(1)$ & $1.321(3)$ \\
$\mathrm{C}(2)-\mathrm{N}(1)$ & $1.384(4)$ & $1.379(1)$ & $1.341(3)$ \\
$\mathrm{N}(1)-\mathrm{C}(6)$ & $1.348(4)$ & $1.362(1)$ & $1.332(3)$ \\
$\mathrm{N}(1)-\mathrm{H}(1)$ & $0.92(3)$ & 1.04 & 1.06 \\
$\mathrm{O}(1 \mathrm{~B}) \cdots \mathrm{H}(1)$ & $1.88(4)$ & 1.78 & 1.69 \\
$\mathrm{O}(1)-\mathrm{H}(2)$ & & & \\
$\mathrm{N}(1) \cdots \mathrm{H}(2 \mathrm{~B})$ & & & \\
\hline
\end{tabular}

${ }^{\text {a }}$ Average distances

The crystal packing of 7 is shown in Figure 3.

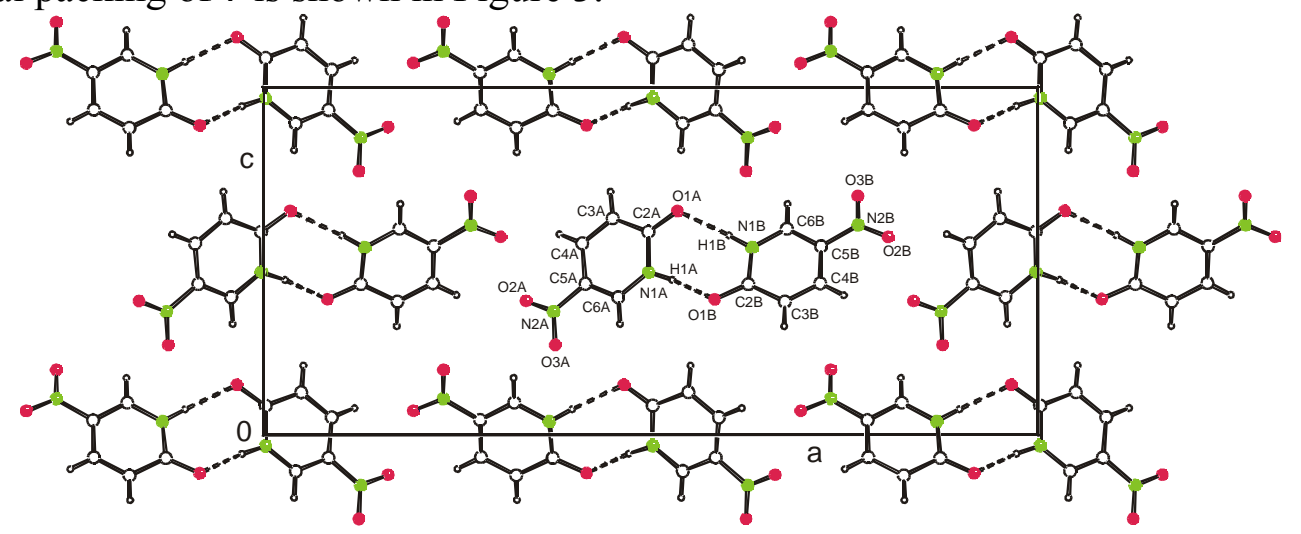

Figure 3. View down the $b$ axis of the crystal packing of 2-hydroxy-5-nitropyridine (7). 


\section{Conclusions}

The main conclusions of the present investigation may be summarized as follows:

i) The electronic effect of substituents bonded at position 6 of the 2-hydroxypyridine derivatives is inductive in character and strongly influences the position of the tautomeric equilibrium. The electronic effect probably involves a balance of the acidity of the N-H group of form $\mathbf{B}$ and of the O-H group of form $\mathbf{A}$.

ii) A consequence of the electron-withdrawing effect is that 2-hydroxy-6-chloropyridine and 2hydroxy-6-methoxypyridine are mainly in the "aromatic form" A, while 2-hydroxypyridine and 2-hydroxy-6-methylpyridine are in form $\mathbf{B}$.

iii) The role of the solvent in affecting the position of the tautomeric equilibrium is connected with its polarity, while the proticity of the solvent appears to be unimportant.

iv) X-Ray diffraction indicates that 2-hydroxy-5-nitropyridine, in the crystal, is in the oxo form B, while in solution both forms may be present.

\section{Experimental Section}

General Procedures. ${ }^{1} \mathrm{H}$ - and ${ }^{13} \mathrm{C}$ - NMR spectra were recorded on a Varian Gemini spectrometer at 300- and $75.46 \mathrm{MHz}$, respectively. Tables 7 and 8 report the main NMR spectroscopic data. Chemical shifts are referred to solvent peaks, and are unaffected by the concentration of 2-hydroxypyridines in the range $1 \times 10^{-2}$ to $1 \times 10^{-3} \mathrm{~mol} \mathrm{dm}^{-3}$. $J$ values are given in Hz. The different NOE measurements were carried out in solutions purged of dissolved oxygen using a nitrogen stream, by pre-saturating the signal for about $10 \mathrm{~s}$ and acquiring the spectrum with the decoupler turned off. The various lines of the multiplets were saturated by cycling the irradiation frequencies about 40 times. A program which accumulates the difference between the two FIDs (that being irradiated and that acquired with the irradiation frequency kept away from any signal) was employed. The control spectrum was subsequently acquired with half the number of scans.

UV/Vis spectra were recorded with Perkin-Elmer Lambda 5 and Lambda 12 spectrophotometers with a thermostatic bath $\left( \pm 0.05^{\circ} \mathrm{C}\right)$. Table 9 reports selected $\mathrm{UV} / \mathrm{Vis}$ spectroscopic data.

Materials. Pyridine derivatives were prepared and purified by the reported procedures. ${ }^{27}$ Solvents were commercial samples (Carlo Erba, RPE) purified by the usual procedures. ${ }^{28}$

Determination of $[B] /[A]$ ratios (by UV/Vis spectrophotometry) were performed by the usual procedures, ${ }^{14,15}$ by using the molar extinction coefficients of fixed NMe and OMe parents.

The solute concentration values for determining $\Delta \mathrm{H}$ and $\Delta \mathrm{S}$ values were appropriate to having $[\mathbf{B}] /[\mathbf{A}]$ ratios close to the value $=1$. 
X-ray data collection and structure refinement. A suitable crystal of 2-hydroxy-5nitropyridine (7) was mounted on a glass fiber in air on a Bruker-AXS SMART CCD area detector diffractometer. The detector to crystal distance was $5 \mathrm{~cm}$. The diffraction experiment was carried out at room temperature. The initial cell parameters and an orientation matrix were obtained from least-squares refinement on reflections measured in three different sets of 20 frames each, in the range $-15<\vartheta<15^{\circ}$. The intensity data comprising a hemisphere were collected using the $\omega$-scan technique with frame width set at $0.3^{\circ}$. The first 50 frames were remeasured at the end of data collection to monitor decay. The collected frames were then processed for integration by software SAINT and an empirical absorption correction was applied by SADABS. ${ }^{29}$ The structure was solved by direct methods (SIR97) ${ }^{30}$ and refined by full-matrix least-squares on $\mathrm{F}^{2}$ using SHELXTL. ${ }^{31}$ Anisotropic displacement parameters were assigned to all non-hydrogen atoms in the structure. All hydrogen atoms were located in the Fourier map but added in calculated positions, apart from the hydrogen atom on nitrogen.

Crystallographic studies of 2-hydroxy-5-nitropyridine (7): $\mathrm{C}_{5} \mathrm{H}_{4} \mathrm{~N}_{2} \mathrm{O}_{3}, \quad M=140.10$, orthorhombic, space group $P_{c a 2_{1}}$ (No. 29), $a=25.930(2), b=3.8288(3), c=11.6589(7) \AA, V=$ 1157.5(1) $\AA^{3}, T=293(2){ }^{\circ} \mathrm{K}, Z=8, \mathrm{D}_{\text {calcd }}=1.608 \mathrm{mg} / \mathrm{m}^{3}, \mu\left(\mathrm{Mo}-\mathrm{K}_{\alpha}\right)=0.136 \mathrm{~mm}^{-1}, 10094$ reflections collected, 2048 unique which were used in all calculations. The value of the goodness of fit indicator was 1.073. Final $R 1(F)=0.0417[\mathrm{I}>2 \sigma(\mathrm{I})]$ and $w R 2\left(F^{2}\right)=0.1045$ (all data). The crystallographic data for 2-hydroxy-5-nitropyridine (7) have been deposited with the Cambridge Crystallographic Data Centre as supplementary publication numbers CCDC 194635. Copies of the data can be obtained, free of charge, on application to CCDC, 12 Union Road, Cambridge, CB2 1EZ, UK [fax: +44(0)-1223-336033 or e-mail: deposit@ccdc.cam.ac.uk]. 
Table 7. ${ }^{1} \mathrm{H}$ NMR spectral data of some 2-hydroxypyridines and of methyl "fixed parents" in different solvents

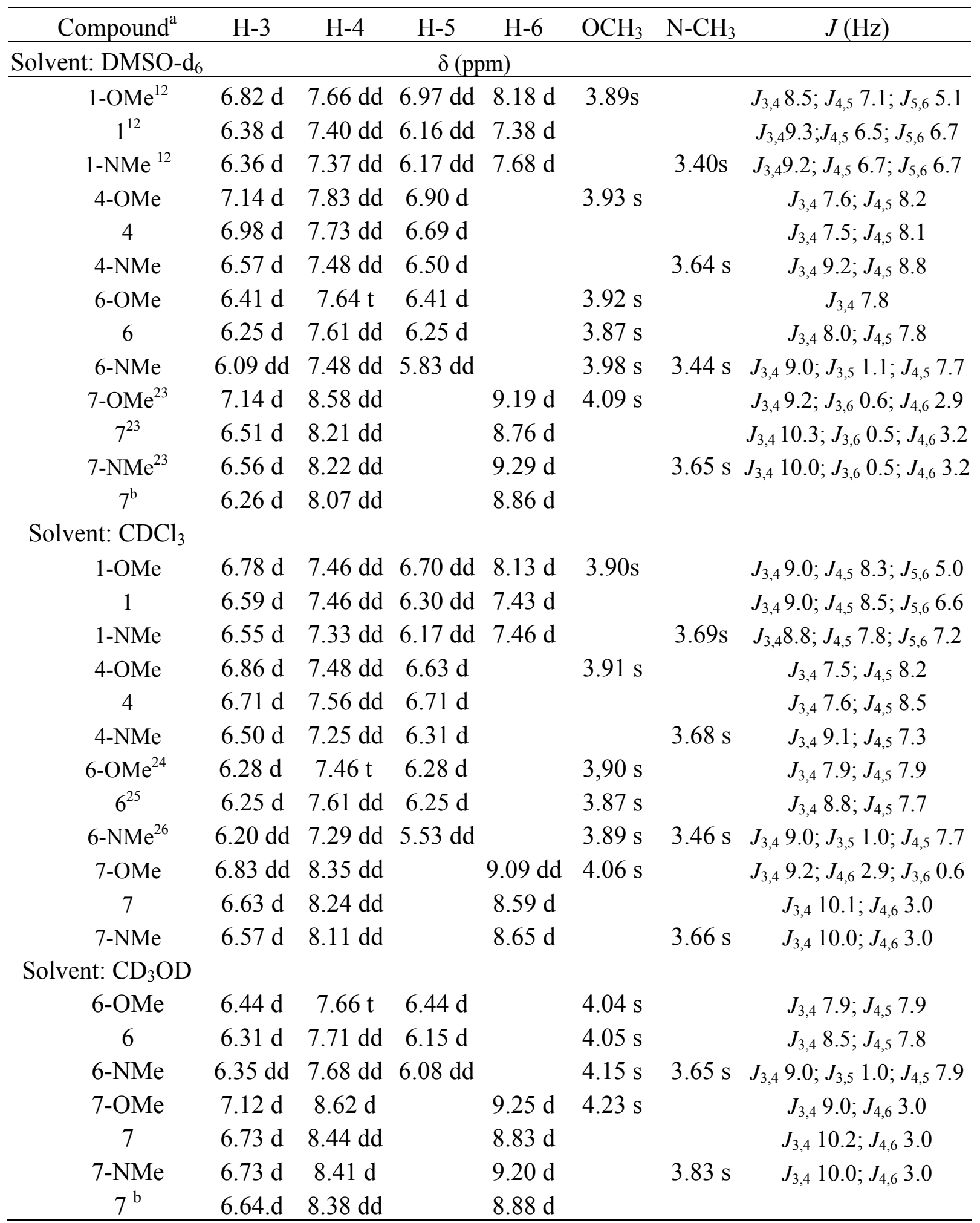

${ }^{\mathrm{a}}$ [Hydroxypyridines] values from $2 \times 10^{-3}$ to $1 \times 10^{-2} \mathrm{~mol} \mathrm{dm}^{-3} \cdot{ }^{\mathrm{b}}$ In the presence of DABCO, [7] $=5 \times 10^{-3} ;[\mathrm{DABCO}]=1 \times 10^{-2} \mathrm{~mol} \mathrm{dm}^{-3}$. 
Table 8. ${ }^{13} \mathrm{C}$ NMR spectral data of some 2-hydroxypyridines and of methyl "fixed parents" in different solvents

\begin{tabular}{|c|c|c|c|c|c|c|c|}
\hline Compound ${ }^{\mathrm{a}}$ & $\mathrm{C}-2$ & $\mathrm{C}-3$ & C-4 & $\mathrm{C}-5$ & C-6 & $\mathrm{OCH}_{3}$ & $\mathrm{~N}-\mathrm{CH}_{3}$ \\
\hline Solvent: DMSO- $\mathrm{d}_{6}$ & \multicolumn{7}{|c|}{$\delta(\mathrm{ppm})$} \\
\hline $1-\mathrm{OMe}^{12}$ & 163.11 & 110.48 & 138.73 & 116.69 & 146.61 & 52.82 & \\
\hline $1^{12}$ & 162.32 & 119.77 & 140.81 & 104.80 & 135.19 & & \\
\hline $1-\mathrm{NMe}^{12}$ & 161.80 & 119.10 & 139.50 & 104.80 & 139.50 & & 41.80 \\
\hline 4-OMe & 163.29 & 109.11 & 141.59 & 116.28 & 147.24 & 53.62 & \\
\hline 4 & 163.71 & 108.48 & 142.10 & 114.81 & 146.98 & & \\
\hline 4-NMe & 161.99 & 106.11 & 139.14 & 117.30 & 137.62 & & 32.68 \\
\hline 6-OMe & 162.66 & 100.83 & 141.21 & 100.83 & 162.66 & 52.75 & \\
\hline 6 & 162.34 & 98.38 & 141.55 & 101.24 & 162.55 & 53.22 & \\
\hline 6-NMe & 157.73 & 83.76 & 139.97 & 109.43 & 161.52 & 56.95 & 27.17 \\
\hline $7-\mathrm{OMe}^{23}$ & 166.86 & 111.20 & 139.41 & 134.54 & 144.55 & 54.74 & \\
\hline $7^{23}$ & 162.10 & 119.02 & 134.28 & 130.15 & 138.74 & & \\
\hline $7-\mathrm{NMe}^{23}$ & 161.47 & 117.60 & 133.37 & 129.53 & 142.60 & & 37.70 \\
\hline $7^{\mathrm{b}}$ & 168.01 & 115.90 & 133.00 & 129.71 & 143.80 & & \\
\hline \multicolumn{8}{|l|}{ Solvent: $\mathrm{CDCl}_{3}$} \\
\hline 1-OMe & 163.80 & 110.62 & 138.03 & 116.23 & 146.55 & 52.79 & \\
\hline 1 & 165.27 & 119.95 & 141.52 & 106.72 & 134.55 & & \\
\hline $1-\mathrm{NMe}$ & 162.23 & 119.23 & 138.90 & 105.20 & 137.88 & & 36.66 \\
\hline $4-\mathrm{OMe}$ & 163.60 & 108.84 & 140.29 & 115.96 & 148.14 & 53.66 & \\
\hline 4 & 164.97 & 111.61 & 142.19 & 113.30 & 144.07 & & \\
\hline 4-NMe & 163.13 & 106.46 & 138.39 & 110.02 & 138.07 & & 33.08 \\
\hline 6-OMe & 163.08 & 100.81 & 140.78 & 100.81 & 163.08 & 53.23 & \\
\hline 6 & 158.77 & 87.02 & 142.86 & 109.39 & 165.07 & 55.57 & \\
\hline 6-NMe & 157.65 & 83.75 & 139.43 & 110.72 & 162.96 & 56.37 & 27.67 \\
\hline 7-OMe & 167.40 & 111.20 & 139.52 & 133.81 & 144.80 & 54.80 & \\
\hline 7 & 164.29 & 120.01 & 135.42 & 132.11 & 136.01 & & \\
\hline 7-NMe & 161.81 & 119.20 & 133.31 & 130.52 & 140.00 & & 38.71 \\
\hline \multicolumn{8}{|l|}{ Solvent: $\mathrm{CD}_{3} \mathrm{OD}$} \\
\hline 6-OMe & 164.84 & 102.18 & 142.42 & 102.18 & 164.84 & 53.95 & \\
\hline 6 & 162.33 & 98.40 & 141.54 & 101.24 & 162.50 & 53.22 & \\
\hline 6-NMe & 159.75 & 87.36 & 142.74 & 110.68 & 165.37 & 57.98 & 28.69 \\
\hline 7-OMe & 169.16 & 112.66 & 135.53 & 141.36 & 145.92 & 50.16 & \\
\hline 7 & 165.18 & 120.59 & 136.49 & 133.21 & 138.62 & & \\
\hline 7-NMe & 164.66 & 119.40 & 135.38 & 132.65 & 142.84 & & 39.10 \\
\hline $7^{b}$ & 168.20 & 119.01 & 135.90 & 133.42 & 141.30 & & \\
\hline
\end{tabular}

${ }^{\mathrm{a}}$ [Hydroxypyridines] values from $2 \times 10^{-3}$ to $1 \times 10^{-2} \mathrm{~mol} \mathrm{dm}^{-3} .{ }^{\mathrm{b}}$ In the presence of DABCO, [7] $=5 \times 10^{-3} ;[\mathrm{DABCO}]=1 \times 10^{-2} \mathrm{~mol} \mathrm{dm}^{-3}$. 
Table 9. $\lambda_{\max }(\mathrm{nm})$ values of the considered 2-hydroxypyridines and related fixed parents in various solvents; $\varepsilon \times 10^{2}\left(\mathrm{dm}^{3} \mathrm{~mol}^{-1} \mathrm{~cm}^{-1}\right)$

\begin{tabular}{llllllllllllll}
\hline Solvent & \multicolumn{2}{c}{ THF } & \multicolumn{2}{c}{$\mathrm{CH}_{2} \mathrm{Cl}_{2}$} & \multicolumn{2}{c}{$\mathrm{DMSO}$} & \multicolumn{2}{c}{$\mathrm{CCl}_{4}$} & \multicolumn{2}{c}{$\mathrm{CH}_{3} \mathrm{OH}$} & \multicolumn{3}{c}{$\mathrm{H}_{2} \mathrm{O}$} \\
\hline Compound & $\lambda_{\max }$ & $\varepsilon$ & $\lambda_{\max }$ & $\varepsilon$ & $\lambda_{\max }$ & $\varepsilon$ & $\lambda_{\max }$ & $\varepsilon$ & $\lambda_{\max }$ & $\varepsilon$ & $\lambda_{\max }$ & $\varepsilon$ \\
\hline 1-OMe & 272 & 34.6 & & & 272 & 58.7 & 274 & 41.0 & 271 & 42.3 & 270 & 42.2 \\
1 & 306 & 46.1 & & & 306 & 58.6 & 299 & 34.3 & 298 & 54.1 & 294 & 51.5 \\
1-NMe & 306 & 47.3 & & & 314 & 58.8 & 308 & 47.9 & 301 & 51.0 & 297 & 52.4 \\
6-OMe & 279 & 67.4 & & & 280 & 62.5 & 282 & 71.6 & 278 & 76.1 & 278 & 75.1 \\
6 & 280 & 62.7 & & & 282 & 69.3 & 281 & 66.8 & 308 & 61.0 & 305 & 75.4 \\
6-NMe & 313 & 79.7 & & & 315 & 71.0 & 316 & 77.4 & 309 & 71.5 & 306 & 74.8 \\
4-OMe & 276 & 62.1 & & & 277 & 64.6 & 278 & 56.4 & 276 & 59.2 & 276 & 62.6 \\
4 & 278 & 63.2 & & & 279 & 66.1 & 278 & 61.4 & 279 & 51.0 & 303 & 58.1 \\
4-NMe & 313 & 63.0 & & & 313 & 65.4 & 315 & 63.1 & 311 & 60.1 & 308 & 63.1 \\
7-OMe & 296 & 99.4 & 299 & 108 & 301 & 121 & 276 & 107 & 293 & 108 & 300 & 96.0 \\
7 & 309 & 98.2 & 305 & 104 & a & & 276 & 109 & 304 & 110 & 301 & 100 \\
7-NMe & 315 & 107 & 307 & 104 & 344 & 90.7 & 276 & 107 & 306 & 90.9 & 303 & 109 \\
\hline
\end{tabular}

${ }^{\mathrm{a}}$ See text.

\section{Acknowledgments}

The authors thank the Ministero dell'Universita' e della Ricerca Scientifica e Tecnologica, the Consiglio Nazionale delle Ricerche (CNR, Roma) and the University of Bologna (Funds for Selected Research Topics, 2000-2002).

\section{References}

1. Forlani, L. Targets in Heterocyclic Systems; Attanasi, O. A.; Spinelli, D. Eds, Italian Society of Chemistry: Roma, 1997; 1, 75.

2. Forlani, L.; Mezzina, E.; Boga C.; Forconi, M. Eur. J. Org. Chem. 2001, 2779.

3. Annese, M.; Bonamartini Corradi, A.; Forlani, L.; Rizzoli, C.; Sgarabotto, P. J. Chem. Soc., Perkin Trans. 1 1994, 615.

4. Forlani, L. J. Heterocyclic Chem. 1992, 29, 1461.

5. Boga, C.; Corradi, A. B.; Forlani, L.; Modarelli, V.; Righi, L.; Sgarabotto, P.; Todesco, P. E. Eur. J. Org. Chem. 2001, 1175 and references cited therein.

6. Boga, C.; Bonamartini, A. C.; Forlani, L.; Modarelli, V.; Righi, L.; Sgarabotto, P. E. J. Chem. Res. (S) 2001, 505; (M) 2001, 1217. 
7. Elguero, J.; Marzin, C.; Katritzky, A. R.; Linda, P. The Tautomerism of Heterocycles: Academic Press: London, 1976.

8. Chevrier, M.; Bensaude, O.; Guillerez, G.; Dubois, J-E. Tetrahedron Lett. 1980, 21, 3359.

9. Chevrier, M.; Guillerez, G.; Dubois, J-E. J. Chem. Soc., Perkin Trans. 2 1983, 979.

10. Bensaude, O.; Chevrier, M.; Dubois, J-E. J. Am. Chem. Soc. 1978, 100, 7055.

11. Bensaude, O.; Dubois, J-E. C. R. Acad. Sci. Paris 1977, 503.

12. Vogeli, U.; Von Philipsborn, W. Org. Magn. Reson. 1973, 5, 551.

13. (a) Krackov, M. H.; Lee, C. M.; Mautner, H. G. J. Am. Chem. Soc. 1965, 87, 892. (b) Hammes, G. G.; Spivey, H. O. J. Am. Chem. Soc. 1966, 1621. (c) Huisgen, R.; Waltz, H. Chem. Ber. 1956, 89, 2616. (d) Davies, M.; Thoms, D. K. J. Org. Chem. 1956, 60, 767. (e) Hobbs, M. E.; Bates, W. W. J. Am. Chem. Soc. 1952, 74, 746.

14. Fujimoto, A.; Inozuka, K. Bull. Chem. Soc. Jpn. 1990, 63, 2292.

15. Frank, J.; Katritzky, A. R. J. Chem. Soc., Perkin Trans. 2 1976, 1428.

16. Exner, O. In Advances in Linear Free Energy Relationships; Chapman, N. B.; Shorter, J., Eds.; Plenum Press; New York, 1972.

17. Charton. M. J. Am. Chem. Soc. 1964, 86, 2033.

18. Forlani, L.; Breviglieri, G.; De Maria, P. J. Chem. Soc., Perkin Trans. 2 1979, 163.

19. Reinheimer, D.; Gerig, J. T.; Garst, R.; Schrier, B. J. Am. Chem. Soc. 1962, 84, 2770.

20. Tokay, N.; Ogretir, C. Theochem 2002, 594, 185 and refs. therein.

21. Yang, H. W.; Craven, B. M. Acta Crystallogr. 1998, B54, 912, and refs. therein.

22. (a) Kvick, A.; Booles, S. S. Acta Crystallogr. 1972, B28, 3405. (b) Kvick, A.; Olovsson, I. Arkiv. Kemi, 1969, 30, 71.

23. Barlin, G. B.; Fenn, M. D. Heterocycles 1986, 24, 1301-1309.

24. Testaferri, L.; Tiecco, M.; Tingoli, M.; Batoli, D.; Massoli, A. Tetrahedron 1985, 41, 1373.

25. Spinner, E.; Yeoh, G. B. J. Chem. Soc. (B) 1971, 279.

26. Nesnow, S.; Shapiro, R. J. Org. Chem. 1969, 34, 2011.

27. (a) Dimroth, K.; Reichardt, C.; Siepmann, T.; Bohlmann, F. Annalen der Chemie 1963, 661, 1. (b) Kuzuya, M.; Noguchi, A.; Kamiya S.; Okuda, T. Chem. Pharm. Bull. 1984, 33(6), 2313. (c) Spinner E.; Yeoh, G. B J. Chem. Soc. (B) 1971, 279. (d) Bouey-Bencteux, E.; Houssin, R.; Dogimont, C.; Hénichart J-P. Heterocycles 1998, 12, 2643. (e) Barlin, G. B.; Pfleiderer, W. J. Chem. Soc. (B) 1971, 1425.

28. Riddick, J. A.; Bunger, W. B. Organic Solvents; Weissberger, A.; Ed. Wiley-Interscience: New York, 1970.

29. Sheldrick, G. M. SADABS, Program for Empirical Absorption Correction, University of Göttingen, Germany, 1996.

30. Altomare, A.; Cascarano, C; Giacovazzo, C.; Guagliardi, A.; Moliterni, A. G. G.; Burna, M. C.; Polidori, G.; Camalli, M.; Spagna, R. SIR97: A New Tool for Crystal Structure Determination and Refinement. J. Appl. Crystallogr. 1999, 32, 115.

31. Sheldrick, G. M. SHELXTLplus Version 5.1 (Windows NT Version) Structure Determination Package; Bruker Analytical X-ray Instruments Inc., Madison, WI, 1998. 